\section{Gastos das famílias brasileiras com medicamentos segundo a renda familiar: análise da Pesquisa de Orçamentos Familiares de 2002-2003 e de 2008-2009}

\author{
Brazilian family spending on medicines: an analysis \\ of data from the Family Budget Surveys, \\ 2002-2003 and 2008-2009
}

\section{Gasto de las familias brasileñas con medicamentos: análisis de encuestas de presupuestos familiares 2002-2003 y 2008-2009}

Leila Posenato Garcia 1,2 Ana Cláudia Sant'Anna 1 Luís Carlos Garcia de Magalhães 1,3 Lúcia Rolim Santana de Freitas 1,4 Adriana Pacheco Aurea 1,5

\title{
Resumo
}

${ }^{1}$ Instituto de Pesquisa Econômica Aplicada, Brasília, Brasil.

2 Secretaria de Vigilância em Saúde, Ministério da Saúde, Brasília, Brasil.

3 Câmara dos Deputados, Brasília, Brasil.

4 Programa de Pós-graduação em Medicina Tropical,

Universidade de Brasília, Brasília, Brasil.

${ }_{5}$ Secretaria Executiva Ministério da Saúde, Brasília, Brasil.

Correspondência L. P. Garcia Instituto de Pesquisa Econômica Aplicada. SBS Quadra 1 Bloco J, Brasília, DF 70076-900, Brasil. leilapgarcia@gmail.com

\begin{abstract}
This study aimed to investigate spending on
Abstract medicines by Brazilian families and related income inequalities, according to types of medicines. A cross-sectional study used data from the Family Budget Surveys conducted in 2002-2003 and 2008-2009. Expenditures were corrected according to the Extended National Consumer Price Index (IPCA). The Concentration Index (CI) was calculated as a measure of inequality. Average monthly spending on medicines was BRL 53.54 in the 2002-2003 survey and BRL 59.02 in 2008-2009. CI showed spending concentration in higher-income families. Spending composition varied according to family income. Lower-income families spent predominantly on analgesics, cold medicines, and anti-inflammatory drugs. Higher-income families concentrated their spending on medicines for diabetes and hypertension (and other cardiovascular diseases). From one survey to the next, even though lower-income households reduced the percentage of their budget spent on medicines, the latter still consume a large proportion of their health spending.
\end{abstract}

Drug Utilization; Health Economics; Health Inequalities; Family
O estudo teve como objetivos investigar os gastos das famílias brasileiras com medicamentos e as desigualdades de renda nestes gastos, segundo categorias de medicamentos. Trata-se de estudo transversal realizado com dados das Pesquisas de Orçamentos Familiares (POF) realizadas em 2002-2003 e 2008-2009. Os valores foram corrigidos pelo Índice Nacional de Preços ao Consumidor Amplo (IPCA). O Índice de Concentração (IC) foi calculado como medida de desigualdade. $O$ gasto médio com medicamentos para o total das famílias correspondeu a $R \$ 53,54$ na POF 20022003 e $R \$$ 59,02 na POF 2008-2009. Os IC revelaram concentração dos gastos entre as famílias de maior renda. A composição destes gastos é diferente conforme a renda das famílias. Entre as de menor renda predominam os gastos com analgésicos, antigripais e anti-inflamatórios. Entre as de maior renda predominam os gastos com medicamentos para diabetes, hipertensão e doenças do coração. Para as famílias de menor renda, apesar da redução do peso do gasto com medicamentos sobre sua renda, este ainda é o principal componente dos gastos com saúde.

Uso de Medicamentos; Economia da Saúde; Desigualdades em Saúde; Família 


\section{Introdução}

O Sistema Único de Saúde (SUS), instituído na Constituição Federal de 1988, tem como diretrizes a universalização do acesso com equidade e a integralidade das ações e serviços de saúde. A execução de ações de assistência terapêutica integral, inclusive farmacêutica, está prevista na Lei no 8.080, de 1990, que regulamenta o SUS 1. Entretanto, apenas em 1998 foi aprovada a Política Nacional de Medicamentos (PNM), com o propósito de garantir a segurança, eficácia e qualidade dos medicamentos, a promoção do uso racional e o acesso da população àqueles considerados essenciais 2 . A assistência farmacêutica no SUS foi consolidada, em 2004, com a aprovação da Política Nacional de Assistência Farmacêutica, atualmente em vigor ${ }^{3}$.

$\mathrm{O}$ acesso a medicamentos no Brasil pode ser estudado por meio de diferentes recortes analíticos, que possibilitam avaliar a importância da assistência farmacêutica do SUS. Um desses recortes é o gasto das famílias com medicamentos. É amplamente reconhecido o peso desses gastos em relação à renda das famílias brasileiras 4,5,6,7. Entretanto, poucos estudos investigaram a evolução temporal desses gastos, bem como a evolução das desigualdades nesses gastos, conforme a renda das famílias.

Os gastos com saúde oneram importante parcela dos orçamentos familiares e governamentais. Na Pesquisa de Orçamentos Familiares (POF) de 2002-2003, verificou-se que os gastos com medicamentos e planos de saúde representaram a principal parcela dos gastos com saúde, em todas as Unidades da Federação, respondendo por cerca de $60 \%$ do total no Brasil e mais de $80 \%$ nas regiões Norte e Nordeste 6 .

Os primeiros trabalhos sobre gastos com saúde da população brasileira, que utilizaram dados das POF, encontram-se compilados em publicações do Instituto de Pesquisa Econômica Aplicada (IPEA) 8,9. Estes estudos apontaram que a renda é fator determinante dos gastos familiares com saúde, ou seja, quanto maior a renda, maior o gasto absoluto com saúde. Por outro lado, são as famílias com menor renda que comprometem, proporcionalmente, maior parte de sua renda com esses gastos. Enquanto, entre as famílias de maior renda, a maior parte do gasto com saúde é destinada aos planos de saúde, entre as famílias de menor renda, os medicamentos representam a maior parcela desses gastos.

$\mathrm{O}$ acesso a medicamentos tem efeitos importantes sobre a situação de saúde individual. A situação de saúde dos indivíduos está relacionada à sua capacidade produtiva e renda. Segundo Andrade \& Lisboa 5, as decisões individuais ou políticas públicas que afetam direta ou indiretamente a saúde têm efeitos cruciais sobre o desenvolvimento econômico de um país. Nesse sentido, a oferta de serviços de saúde e a situação de saúde da população aparecem como preocupações crescentes entre os formuladores de políticas públicas. $\mathrm{O}$ acesso a medicamentos também é um indicador da qualidade e resolutividade do sistema de saúde e um determinante importante do cumprimento do tratamento prescrito 10 .

No Brasil, o componente privado tem papel relevante no gasto total com saúde. As famílias brasileiras têm participação fundamental no financiamento da assistência à saúde 11. Existem poucos estudos na literatura nacional e internacional que analisaram os gastos privados com saúde 5 . Assim, é importante estudar os gastos das famílias com medicamentos e mensurar o impacto desses gastos sobre a renda familiar. Essas informações podem fornecer subsídios para avaliar a eficiência e eficácia dos programas de assistência farmacêutica do SUS.

O presente estudo tem como objetivos estimar os gastos das famílias brasileiras com medicamentos, investigar as desigualdades de renda nesses gastos, segundo as categorias de medicamentos e descrever sua evolução nas POF realizadas em 2002-2003 e 2008-2009.

\section{Métodos}

As fontes do presente estudo são os microdados das POF, realizadas pelo Instituto Brasileiro de Geografia e Estatística (IBGE), em 2002-2003 e 2008-2009. Estas pesquisas revelam informações detalhadas sobre tipos de gastos, quantidade, valor, especialidade de serviços, assim como sobre os rendimentos das famílias ou unidades de consumo. Estas são definidas como morador ou conjunto de moradores que compartilham o mesmo estoque de alimentos e/ou conjunto de despesas alimentares. Quando não existem estoques, nem despesas com alimentos, a unidade de consumo é definida perante as despesas com moradia 12 .

As famílias são consideradas como unidade de análise das POF. Isso é importante, pois, muitas vezes, as despesas registradas para um morador podem se referir a produtos comprados para outro morador, ou mesmo para um não residente. Assim, por exemplo, as despesas com a compra de medicamentos para crianças provavelmente serão registradas na conta de um de seus pais.

Os gastos com saúde nas POF são captados segundo categorias distintas, sendo que uma dessas categorias corresponde aos medicamentos (remédios). O gasto com medicamentos é 
coletado por meio do questionário de despesa individual, no período de trinta dias anteriores à entrevista. São registrados os gastos com medicamentos em geral, independentemente de serem ou não de uso regular ${ }^{4}$. É possível agregar os gastos com medicamentos em algumas categorias, determinadas nas POF.

Para o presente estudo, devido a diferenças nos questionários, foi necessário compatibilizar as informações para compor categorias comparáveis entre as POF estudadas. Para as análises dos gastos com cada categoria de medicamento, foram realizadas as seguintes classificações e procedimentos para compatibilização:

- Diabetes: para diabetes, nas duas edições das POF;

- Hipertensão: vasodilatador e para pressão arterial, nas duas edições da POF;

- Colesterol: redutor de colesterol e para triglicerídeos, nas duas edições da POF;

- Doenças do coração: para insuficiência cardiovascular (POF 2002-2003), para insuficiência cardiovascular e para problemas cardíacos e circulatórios (POF 2008-2009);

- Anticoncepcional: anticoncepcional, nas duas edições da POF;

- Sistema nervoso: para labirintite, calmante, antidepressivo, calmante natural, antiepiléptico, anticonvulsivo, para epilepsia, hidantal, para estresse, antiparksoniano, para sistema nervoso, neurológico, para memória, para tontura, para vertigem, ansiolítico, antipsicótico, para alzheimer, para derrame cerebral e sonífero (POF 20022003), antidepressivo, calmante, para estresse, ansiolítico, calmante natural, sonífero, para sistema nervoso, antiepiléptico, anticonvulsivo, antipsicótico, para Alzheimer, para derrame cerebral, antiparksoniano, neurológico, para memória, para tontura, para circulação, para psicose (POF 2008-2009);

- Para infecções: anti-infeccioso, antibiótico, creme vaginal, remédio ginecológico, para infecções da boca e garganta e pomada vaginal (POF 2002-2003), para infecção, anti-infeccioso, antibiótico, creme vaginal, remédio ginecológico, antibacteriano, pomada vaginal, anti-infeccioso e anti-inflamatório, antivirótico e para herpes (POF 2008-2009);

- Vitaminas: vitaminas e vitamina B12 (POF 2002-2003), vitaminas, vitamina B12, Complexo $\mathrm{B}$, Complexo vitamínico e vitamina C (POF 20082009);

- Anti-inflamatórios: anti-inflamatório e antirreumático, para sinusite, para labirintite, para reumatismo, para coluna e corticoide, nas duas edições da POF;

- Antigripais: antigripal e antitussígeno, antigripal, antitussígeno, antigripal e analgésico, antigripal e antitérmico, antigripal homeopático, expectorante (xarope) e mel medicinal, nas duas edições da POF;

- Analgésicos: analgésico e antitérmico, antitérmico, gelol, para contusão, para enxaqueca, xilocaína, anestésico, analgésico, analgésico e anti-inflamatório (POF 2002-2003), analgésico e antitérmico, antitérmico, para enxaqueca, analgésico, analgésico e anti-inflamatório (POF 20082009);

- Antialérgicos: antialérgico, descongestionante nasal, antialérgico homeopático, descongestionante nasal homeopático, anticongestionante, creme antialérgico e pastilha para garganta (POF 2002-2003), antialérgico, antialérgico homeopático, creme antialérgico e sabonete antialérgico (POF 2008-2009).

Neste estudo, foi considerado apenas o gasto "monetário" com medicamentos, tendo sido desconsideradas todas as formas de obtenção não-monetárias (doação, retirada de negócio, troca e produção própria). Para esclarecimentos sobre as despesas e rendimentos monetários e não-monetários, sugere-se consultar publicação do IBGE 12.

Para permitir a comparação dos valores absolutos dos gastos com medicamentos calculados a partir das duas POF, foi feita correção segundo o Índice Nacional de Preços ao Consumidor Amplo (IPCA), do IBGE (disponível na página de Internet do IPEA Data: http:/ / www.ipeadata.gov. br), tendo como base a data de referência da POF 2002-2003, 15 de janeiro de 2003. O IPCA foi selecionado por ser um índice de referência para famílias residentes em áreas urbanas, dentro do Sistema Nacional de Índices de Preços ao Consumidor (SNIPC), cuja atualização ocorre mediante a POF, bem como por ser um índice já utilizado em outros estudos da área 9 .

Para investigar a dimensão socioeconômica das desigualdades nos gastos com saúde ao longo do tempo, dividiram-se as famílias em quintos de renda familiar mensal per capita. Para os gastos com medicamentos e suas categorias, foram calculados os percentuais de famílias que tiveram gastos positivos e não nulos, os gastos médios das famílias que tiveram registro desse tipo de gasto, bem como a média dos gastos de todas as famílias da amostra, segundo quintos de renda familiar mensal per capita e para o total da amostra. A participação do gasto com cada categoria de medicamento em relação ao gasto total com medicamentos foi calculada como porcentagem, também para o total da amostra e segundo quintos de renda. A razão entre os valores gastos com determinadas categorias de medicamentos pelas famílias pertencentes aos quintos extremos de renda foi calculada dividin- 
do-se o valor médio do gasto com cada categoria de medicamento das famílias pertencentes ao último quinto de renda, $20 \%$ das famílias com maior renda, pelo mesmo valor das famílias pertencentes ao primeiro quinto, $20 \%$ das famílias com menor renda.

O Índice de Concentração (IC) foi calculado, utilizando a renda familiar mensal per capita como indicador socioeconômico, para mensurar as desigualdades no gasto para cada grupo de medicamentos estudado. O IC é uma medida estatística que incorpora dados de toda população e é sensível a alterações na distribuição da população entre grupos socioeconômicos 13 . Suas vantagens são: (i) utilizar informações de todos os estratos sociais; (ii) incorporar dados sobre o tamanho dos estratos populacionais; (iii) não exigir a ordenação dos estratos sociais; (iv) ser válido para acompanhamento ao longo do tempo; e (v) permitir ilustrar graficamente tendências nas desigualdades 14 .

O IC foi calculado por meio da seguinte fórmula 15,16: $\mathrm{IC}=2 * \Sigma\left[\mathrm{f}_{\mathrm{t}}^{*} \mu_{\mathrm{t}}^{*} \mathrm{R}_{\mathrm{t}}\right] / \mu$ - 1 , na qual $\mathrm{f}_{\mathrm{t}}$ é a parcela da população do grupo socioeconômico t a qual o indivíduo pertence; $\mu_{\mathrm{t}}$ é a média do grupo; $\mathrm{R}_{\mathrm{t}}$ é a posição fracional na ordenação socioeconômica e $\mu$ é a média geral. Os erros-padrão, para o cálculo dos intervalos de confiança foram obtidos por meio do método da covariância 15 .

Apesar de ser calculado da mesma maneira que o coeficiente de Gini, o IC pode assumir valores no intervalo $[-1,1]$. Quanto mais próximo de zero, mais próximo à igualdade e quanto mais próximo a -1 ou 1, maior a desigualdade. Na presente análise, o sinal positivo indica concentração do gasto entre famílias com maior renda.

Para a compatibilização dos dados das POF, utilizou-se o software SAS 9.2 (SAS Inst., Cary, Estados Unidos, e para a análise dos dados utilizou-se o Stata 10.1 (Stata Corp., College Station, Estados Unidos), levando em conta a variável fator de expansão disponibilizada pelo IBGE. O cálculo do IC e de seu desvio padrão foi feito no Stata, utilizando o cálculo proposto por Kakwan et al. 15. Os gráficos foram produzidos no software Excel 2010 (Microsoft Corp., Estados Unidos).

$\mathrm{O}$ estudo utilizou dados de fonte secundária disponibilizados pelo IBGE, garantindo o anonimato dos participantes. O presente estudo está em conformidade com as normas que regulamentam as pesquisas envolvendo seres humanos no Brasil.

\section{Resultados}

A amostra da POF de 2002-2003 incluiu 48.568 famílias, com tamanho médio de 3,6 pessoas e renda média de $\mathrm{R} \$ 2.153,86$, em valores de janeiro de 2009 atualizados pelo IPCA. Por sua vez, a amostra da POF 2008-2009 abrangeu 56.091 famílias, com tamanho médio de 3,3 pessoas e renda média de $\mathrm{R} \$ 2.288,77$.

Segundo a última POF, o gasto médio com medicamentos para o total das famílias brasileiras correspondeu a $\mathrm{R} \$ 59,02$ e aquele para as famílias que registraram gasto com esses produtos equivaleu a $\mathrm{R} \$ 76,31$. Segundo a POF anterior, estes gastos corresponderam, respectivamente, a $\mathrm{R} \$ 53,54$ e $\mathrm{R} \$ 71,20$, em valores corrigidos. No período 2002-2009, o gasto das famílias brasileiras com medicamentos teve variação positiva de $10 \%$, enquanto que a proporção de famílias que gastaram com medicamentos permaneceu relativamente estável $(75,2 \%$ e $77,3 \%$ nas POF 2002 2003 e 2008-2009, respectivamente).

A proporção das famílias que tiveram gasto com medicamentos, segundo categorias de medicamentos e quintos de renda familiar, pode ser observada na Tabela 1. Durante o período de referência para o estudo, analgésicos foi a categoria de medicamento com maior proporção de famílias que registraram gasto com medicamentos (35,4\% na POF 2002-2003 e $42 \%$ na POF 2008-2009), seguidos pelos anti-inflamatórios, antigripais e anti-hipertensivos. Além dos analgésicos, no período 2002-2009, houve aumento das proporções de famílias que tiveram gastos com antigripais, medicamentos para colesterol e problemas do coração. As proporções de famílias que tiveram gastos com medicamentos para hipertensão, vitaminas, anticoncepcionais, antialérgicos e medicamentos para diabetes e sistema nervoso permaneceram relativamente estáveis. Entre as famílias de todos os quintos de renda, houve decréscimo no percentual daquelas que tiveram gasto com anti-inflamatórios e anti-infecciosos.

Nota-se, ainda, que existe um gradiente de renda importante para a compra de medicamentos para diabetes, hipertensão, problemas do coração, colesterol, antialérgicos e anticoncepcionais, ou seja, a proporção de famílias que tiveram gasto com esses medicamentos aumenta marcadamente à medida que aumenta a sua renda. Por outro lado, notadamente para os analgésicos e antigripais não existe esse gradiente, ou seja, as proporções de famílias que tiveram gasto com esses medicamentos não se alteram segundo os quintos de renda.

É interessante conhecer quais as proporções que os gastos com determinadas categorias de medicamentos representam em relação ao gasto total com medicamentos. Essas proporções, para o primeiro e o último quintos de renda familiar per capita, bem como para o total das famílias, 
Tabela 1

Percentual das famílias que tiveram gasto com medicamentos *

\begin{tabular}{|c|c|c|c|c|c|c|c|c|c|c|c|c|}
\hline \multirow{2}{*}{$\begin{array}{l}\text { Categorias de } \\
\text { medicamentos }\end{array}$} & \multicolumn{6}{|c|}{ 2002-2003 (quintos) } & \multicolumn{6}{|c|}{ 2008-2009 (quintos) } \\
\hline & 10 & 20 & 30 & 40 & 50 & Brasil & 10 & 20 & 30 & 40 & 50 & Brasi \\
\hline Analgésicos & 31,4 & 35,3 & 34,7 & 37,8 & 37,6 & 35,4 & 41,5 & 43,6 & 41,8 & 41,5 & 41,5 & 42,0 \\
\hline Antigripais & 12,9 & 13,8 & 13,9 & 13,8 & 14,0 & 13,7 & 14,4 & 15,8 & 14,8 & 15,4 & 16,3 & 15,3 \\
\hline Vitaminas & 9,1 & 9,5 & 8,9 & 9,0 & 12,0 & 9,7 & 8,3 & 8,6 & 7,7 & 7,4 & 11,5 & 8,7 \\
\hline Anticoncepcionais & 5,9 & 8,7 & 10,5 & 12,7 & 11,4 & 9,8 & 6,6 & 9,6 & 10,8 & 11,8 & 12,6 & 10,3 \\
\hline Antialérgicos & 4,2 & 5,8 & 7,3 & 8,1 & 11,9 & 7,5 & 3,6 & 6,7 & 6,6 & 7,9 & 9,4 & 6,8 \\
\hline Colesterol & 0,3 & 0,5 & 1,2 & 2,0 & 4,6 & 1,7 & 0,7 & 1,5 & 2,2 & 4,2 & 7,6 & 3,3 \\
\hline Sistema nervoso & 5,7 & 8,6 & 9,4 & 11,9 & 13,1 & 9,8 & 4,7 & 7,4 & 8,8 & 10,5 & 12,6 & 8,8 \\
\hline Diabetes & 0,6 & 1,4 & 2,8 & 3,3 & 3,9 & 2,4 & 1,0 & 1,9 & 3,0 & 3,4 & 6,8 & 3,2 \\
\hline Problemas do coração & 0,4 & 0,9 & 1,9 & 2,1 & 2,3 & 1,5 & 1,7 & 3,5 & 5,3 & 6,3 & 8,0 & 5,0 \\
\hline Anti-inflamatórios & 15,7 & 19,1 & 20,2 & 21,8 & 21,9 & 19,7 & 12,6 & 16,2 & 16,0 & 17,9 & 18,1 & 16,2 \\
\hline Hipertensão & 5,0 & 11,0 & 14,8 & 17,8 & 22,8 & 14,3 & 4,8 & 10,7 & 15,5 & 17,4 & 24,3 & 14,5 \\
\hline Infecções & 9,8 & 11,7 & 11,1 & 12,1 & 11,4 & 11,2 & 7,2 & 8,3 & 7,9 & 7,5 & 9,2 & 8,0 \\
\hline
\end{tabular}

* Durante o período de referência para coleta de dados da respectiva Pesquisa de Orçamentos Familiares, correspondente a trinta dias anteriores à entrevista, para os gastos com medicamentos.

nas POF 2002-2003 e 2008-2009, podem ser observadas na Figura 1.

Na POF 2008-2009, para as famílias de maior renda (último quinto), o gasto com medicamentos para hipertensão, diabetes, problemas do coração e colesterol, representou $26,1 \%$ do gasto total dessas famílias com medicamentos. Para as famílias de menor renda (primeiro quinto), essa proporção representou apenas $10,1 \%$. Por outro lado, essas famílias comprometeram $46,5 \%$ de seu gasto total com medicamentos na aquisição de analgésicos, antigripais, anti-inflamatórios e vitaminas, enquanto que as famílias do último quinto comprometeram $25 \%$.

De maneira a compreender melhor essa situação, a Figura 2 mostra o gasto, com categorias de medicamentos selecionadas, das famílias pertencentes ao primeiro e ao último quintos de renda familiar per capita, nas POF de 2002-2003 e 2008-2009.

Na POF 2008-2009, como categoria individu$\mathrm{al}$, os analgésicos representaram o maior volume de gasto das famílias de menor renda (primeiro quinto). Em seu total, essas famílias gastaram, em média, $\mathrm{R} \$ 4,72$ mensais com a aquisição de analgésicos. Somando-se analgésicos, antigripais e anti-inflamatórios, as famílias no primeiro quinto de renda, gastaram, em média, $\mathrm{R} \$$ 9,78 por mês nas compras desses medicamentos. Por sua vez, as famílias com maior renda (último quinto) gastaram mais com medicamentos para hipertensão e sistema nervoso, R $\$ 12,72$ e $\mathrm{R} \$$ 12,04 , respectivamente. Conforme esperado, as famílias do último quinto registraram um maior valor absoluto de gasto que as famílias do primeiro quinto para todas as categorias de medicamentos ilustradas. De maneira geral, os gastos com medicamentos aumentaram para a maioria das categorias de medicamentos investigadas.

A partir desses valores, calcularam-se as razões entre os valores gastos com determinadas categorias de medicamentos pelas famílias pertencentes aos quintos extremos de renda, que podem ser observadas na Figura 3.

Na POF 2008-2009, para o gasto total com medicamentos, as famílias no último quinto de renda familiar per capita gastaram 4,5 vezes o valor absoluto que as famílias no primeiro quinto gastaram. A maior diferença relativa foi observada para medicamentos para colesterol $(17,1$ vezes), seguido pelos medicamentos para diabetes (15,9 vezes). As menores razões foram observada para analgésicos e antigripais (1,9 vezes e 2,0 vezes o valor gasto pelas famílias pertencentes ao primeiro quinto, respectivamente). $\mathrm{Na} \mathrm{POF}$ 2002-2003, a razão entre o gasto com medicamentos das famílias de maior e menor renda $(4,4$ vezes) foi semelhante à POF 2008-2009. A maior diferença relativa também foi observada para medicamentos para colesterol, porém seu valor (27,6 vezes) foi superior à última POF.

Devido ao fato de que, ao se analisar apenas os quintos extremos de renda, $60 \%$ das famílias são desconsideradas, foram calculados os índices de concentração para o gasto das famílias com medicamentos, bem como determinadas 
Figura 1

Percentual do gasto com categorias de medicamentos no gasto total com medicamentos, no primeiro (10) e último (5Q) quintos de renda familiar per capita e total das famílias. Pesquisa de Orçamentos Familiares 2002-2003 e 2008-2009.

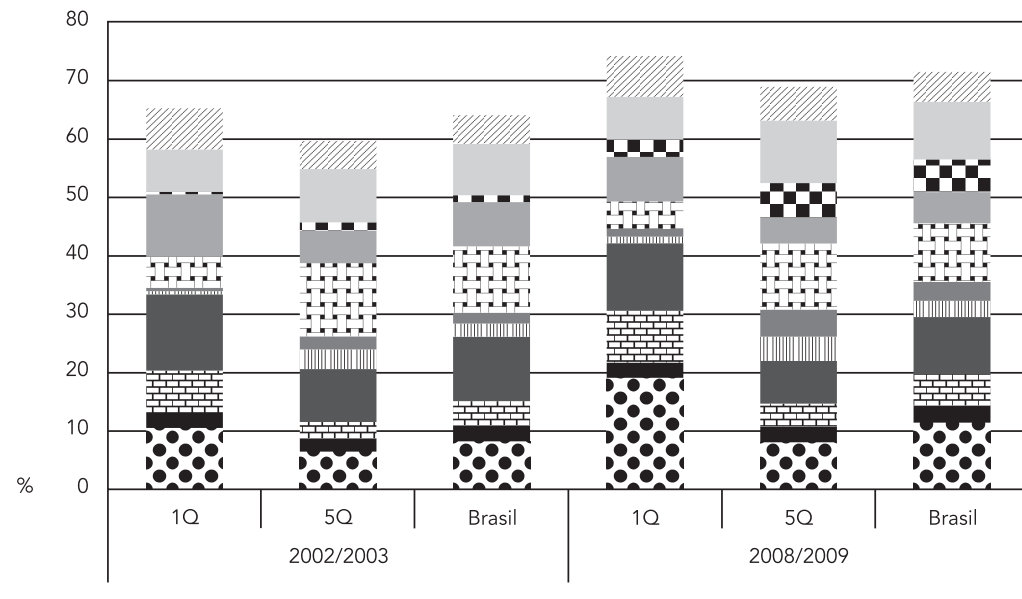

1. Analgésicos

Anticoncepcionais

監 Antigripais

Antiinflamatórios

血 Colesterol

Diabetes

2: Hipertensão

Infecções

17 Problemas do coração

Sistema nervoso

Vitaminas

\section{Figura 2}

Gasto médio mensal familiar em Reais (R\$) corrigidos com categorias de medicamentos, no primeiro (1Q) e último (5Q) quintos de renda familiar per capita e para o total das famílias. Pesquisa de Orçamentos Familiares 2002-2003 e 2008-2009.

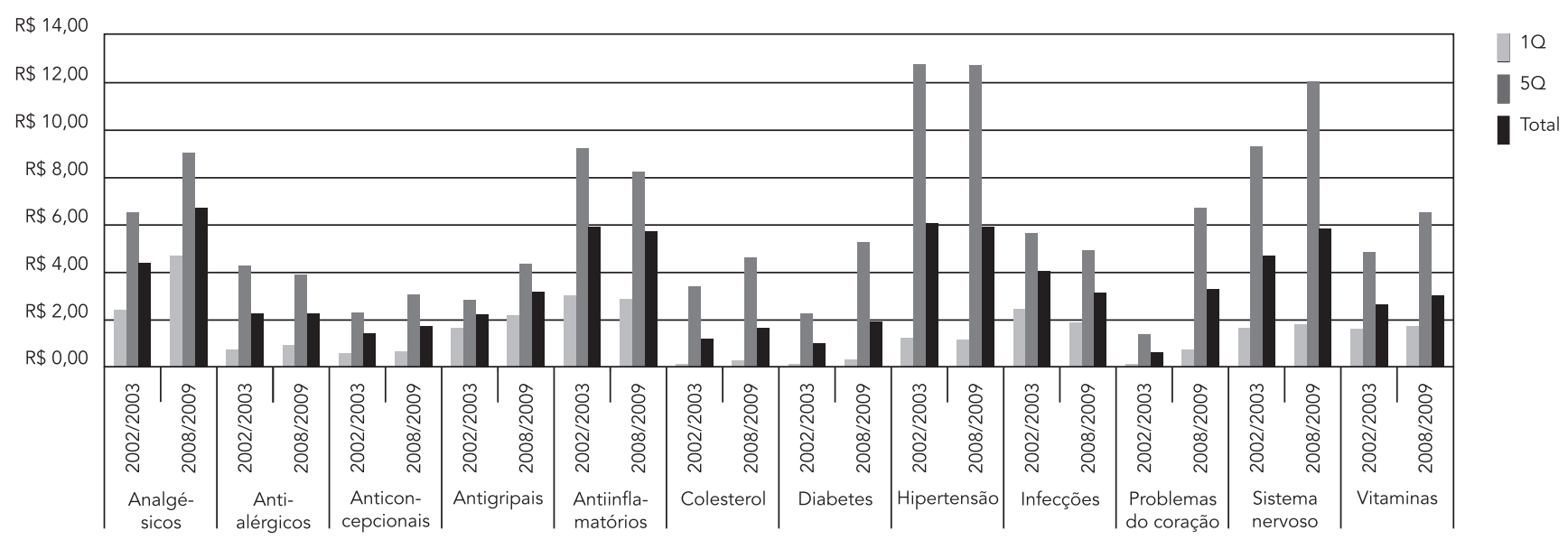




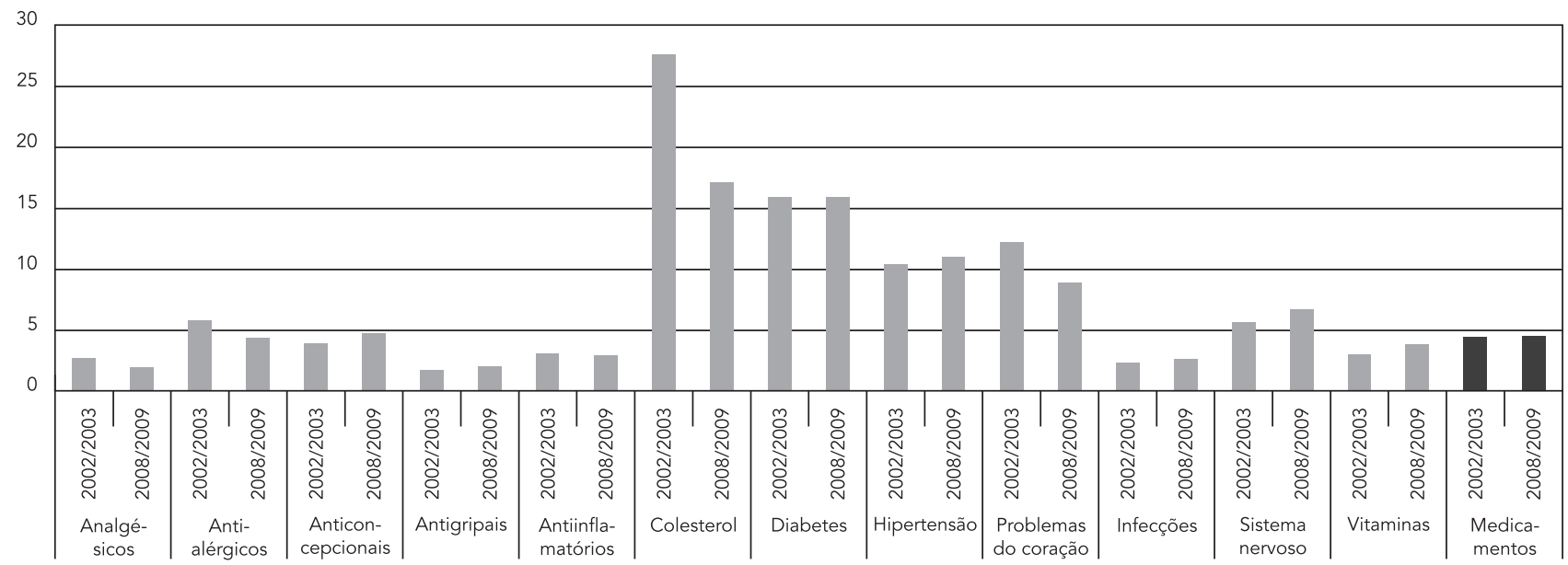

categorias dos mesmos, segundo a renda mensal familiar per capita. Os IC e seus respectivos intervalos de $95 \%$ de confiança, para as POF de 2002-2003 e 2008-2009, podem ser observados na Figura 4.

Todos os IC foram positivos, indicando uma distribuição desproporcional do gasto com medicamentos segundo a renda das famílias, com concentração desse gasto entre as famílias de maior renda. Observa-se maior concentração entre as famílias com maior renda dos gastos com medicamentos para diabetes, doença do coração, colesterol, hipertensão e sistema nervoso, em ordem decrescente do IC.

Essa concentração é conhecida, entretanto, é interessante estudar sua evolução temporal. Não houve diferença estatisticamente significativa entre o IC do gasto com medicamentos, entre as POF 2002-2003 e 2008-2009, indicando estabilidade na desproporção entre o gasto das famílias com maior e menor renda. Tal estabilidade também foi verificada para as categorias específicas de medicamentos, exceto para os antigripais e vitaminas, para os quais houve aumento estatisticamente significativo do IC, ou seja, os gastos com esses medicamentos se tornaram mais concentrados entre as famílias com maior renda.

\section{Discussão}

O presente estudo estimou os gastos das famílias brasileiras com medicamentos e apresentou a magnitude das desigualdades de renda nestes gastos, a partir dos dados de duas POF (20022003 e 2008-2009). Além de atualizar os dados já publicados, incorporando aqueles derivados da POF 2008-2009, foi feito um esforço para compatibilizar as variáveis referentes aos tipos de medicamentos comprados pelas famílias brasileiras, nas duas POF estudadas. Não foram encontrados, na literatura, outros estudos de abrangência nacional que tivessem investigado os gastos com medicamentos das famílias brasileiras, desagregados por categorias de medicamentos. Sobre este aspecto, destaca-se o ineditismo do presente estudo.

Os principais achados são que, apesar da proporção de famílias que relataram gastos com medicamentos ter permanecido relativamente estável, durante os respectivos períodos de referência das POF estudadas, os gastos com medicamentos das famílias brasileiras apresentaram variação relativa positiva de $10 \%$. A composição destes gastos segundo categorias de medicamentos foi bastante diferente conforme a renda das famílias. Enquanto entre as famílias de menor renda predominaram os gastos com analgésicos, antigripais e anti-inflamatórios, entre aquelas de maior renda predominaram os 
Figura 4

Índices de concentração (IC) e respectivos intervalos de $95 \%$ de confiança do gasto das famílias com medicamentos segundo a renda mensal familiar per capita.

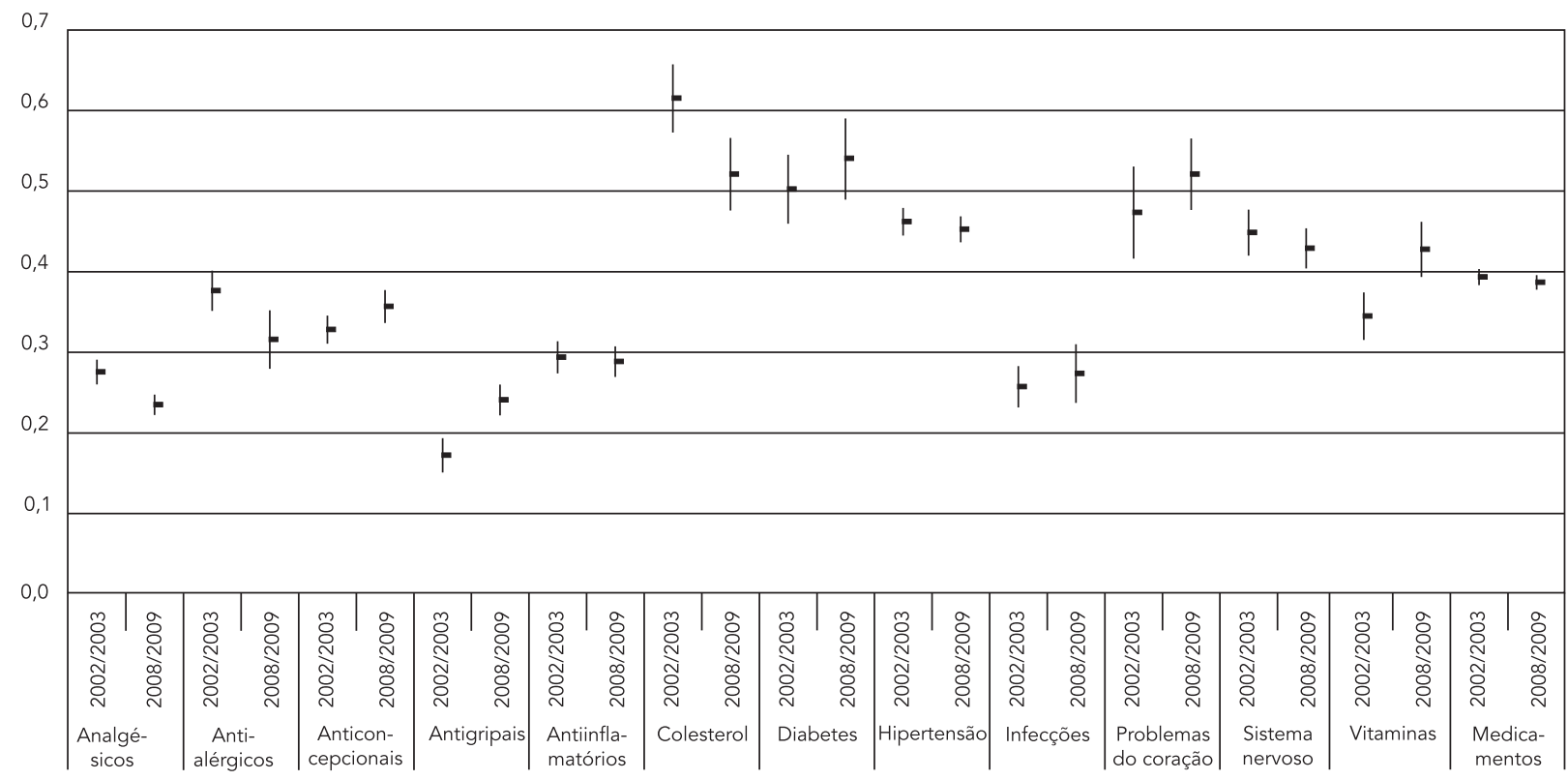

gastos com medicamentos para o tratamento de doenças crônicas, incluindo diabetes, hipertensão e doenças do coração. Conforme esperado, houve forte concentração dos gastos com medicamentos entre as famílias com maior renda. Para a maior parte das categorias de medicamentos estudadas, observou-se estabilidade da participação dos grupos de renda no total do gasto com medicamentos, com manutenção das desigualdades.

Este estudo verificou apenas os gastos monetários das famílias com medicamentos, com as restrições inerentes aos dados das POF. Uma limitação se refere à categorização dos medicamentos. O estudo foi realizado com dados secundários, assim, a classificação foi feita a partir dos dados disponíveis, que não permitem conhecer os medicamentos, nem as formas farmacêuticas que foram compradas. Assim, as categorias definidas apresentam imperfeições. Algumas categorias são muito abrangentes, por exemplo, anti-infecciosos, que abrange medicamentos diversos, como antibióticos, antivirais e antifúngicos. A categoria Sistema Nervoso também agrega medicamentos bastante distintos. Por outro lado, a categoria Antigripais é mais específica, entre- tanto, agrega medicamentos que poderiam estar em outras categorias, como Analgésicos, Antialérgicos ou Anti-inflamatórios. Esse exemplo evidencia a dificuldade em caracterizar medicamentos que contém combinações de princípios ativos. Além disso, essa classificação é feita pelos próprios respondentes da POF, que podem incorrer em erros.

Outra limitação é que não é possível, pelo menos de maneira direta, fazer uma relação entre o gasto com medicamentos e o perfil demográfico e epidemiológico individual, uma vez que as informações sobre gasto são referentes às unidades de consumo (famílias), e não aos indivíduos que consumiram os medicamentos comprados. Também não é possível determinar a finalidade, quantidade ou o tipo específico de medicamento que foi adquirido, nem quais os moradores do domicílio (ou se destinam-se a não residentes) que deles fazem uso 4 .

A consideração apenas das famílias que tiveram registro de gasto pode superestimar a proporção da renda comprometida com os gastos, especialmente quando os períodos de referência para coleta dos dados são curtos, como é o caso dos medicamentos. Quando os períodos de 
referência são mais longos, uma maior proporção das famílias pode contribuir com as médias. Quando se consideram todas as famílias (aquelas que tiveram e que não tiveram gastos) são observadas proporções mais baixas de comprometimento da renda com o gasto em questão, o que é representativo de períodos de tempo mais longos 17. Por outro lado, quando se considera somente as famílias que tiveram gastos positivos, evita-se a diluição do peso do gasto causada pelas famílias com gasto zero 5 . No presente estudo, optou-se por apresentar o gasto médio mensal com medicamentos para o total das famílias. $\mathrm{O}$ IC também foi calculado considerando-se o total das famílias. Dessa maneira também foi calculada a participação percentual das despesas com medicamentos na renda familiar, segundo grupos de renda. Entretanto, para o cálculo das proporções dos gastos com categorias em relação com o gasto total com medicamentos, foram consideradas apenas as famílias que registraram gasto com estes itens.

O padrão desigual de gasto com medicamentos das famílias brasileiras, quando ordenadas pela sua renda, não se alterou substancialmente nas últimas décadas. Diniz et al. 18 constataram, a partir das informações das POF de 1987/1988, 1995/1996 e de 2002/2003, tendência de manutenção e mesmo de aumento da diferença do gasto com medicamentos entre as famílias de maior e menor renda. A compra de medicamentos era o componente de maior peso no total dos gastos com saúde das famílias de menor renda, embora em termos absolutos seja muito menor que o gasto das famílias de maior renda.

Ficou evidente, no presente estudo, que apesar da proporção de famílias que relataram gastos com medicamentos ter permanecido relativamente estável, houve um aumento da proporção de famílias que tiveram gasto com determinadas categorias de medicamentos, principalmente analgésicos, anticoncepcionais e medicamentos para diabetes e problemas do coração. Destaca-se o fato de que, segundo a POF 2008-2009, 42\% das famílias tiveram registro de gasto com analgésicos, no período de referência para coleta de dados da POF, que é de 30 dias para medicamentos. Assim, acredita-se que a proporção de famílias que têm gastos com esse tipo de medicamento seria muito maior, caso os dados tivessem sido coletados durante um período mais longo. A soma dos gastos com analgésicos, antigripais, anti-inflamatórios e vitaminas correspondeu a um quarto do gasto total com medicamentos das famílias, na POF 2008-2009. Entre as famílias de menor renda, pertencentes ao primeiro quinto, esse gasto correspondeu a quase a metade do gasto total com medicamen- tos, sendo o gasto médio com analgésicos superior àquele com medicamentos para diabetes $\mathrm{e}$ colesterol.

Segundo regulamentação da Agência Nacional de Vigilância Sanitária (ANVISA), os analgésicos são medicamentos isentos de prescrição. Dados de 2009 do Sistema Nacional de Informações Tóxico-Farmacológicas (SINITOX; http://www.fiocruz.br/sinitox_novo/cgi/cgilua. exe/sys/start.htm?sid=349, acessado em 11/ Mai/2012) apontaram os medicamentos como responsáveis pelo maior número de intoxicações no Brasil, cerca de $30 \%$ do total de casos. No mesmo ano, ficaram em segundo lugar entre os agentes causadores de óbitos, correspondendo a $17 \%$ do total, atrás apenas dos agrotóxicos de uso agrícola, responsáveis por $42 \%$ dos óbitos. As crianças de um a quatro anos são as que mais se intoxicam com medicamentos. Estudos apontam os analgésicos como importante causa de intoxicação 19,20.

No presente estudo, verificou-se que o gasto das famílias com analgésicos foi elevado (na POF 2008-2009, correspondeu a $11,4 \%$ do gasto com medicamentos). As evidências indicam a necessidade de se intensificar os esforços de promoção do uso racional de medicamentos, com ênfase no esclarecimento sobre os riscos da automedicação. Assim, corrobora-se a iniciativa da ANVISA de revisar a $R D C$ no 138/2003 3, que dispõe sobre o enquadramento na categoria de venda de medicamentos, indicando aqueles que são isentos de prescrição médica.

Quanto aos anti-infecciosos, observou-se redução, tanto do percentual de famílias que tiveram gasto, quanto do valor gasto, no período estudado. Acredita-se que a promoção do uso racional de medicamentos, que faz parte das diretrizes da PNM 2, pode ter contribuído para esse achado, todavia, são necessários estudos para investigar as causas desta redução. Espera-se que, com a proibição da venda de antibióticos sem prescrição, vinculando a dispensação à retenção de receita de controle especial, instituída por meio da $R D C$ no 44/2010 21, substituída pela RDC no 20/2011 22, da ANVISA, o percentual de famílias que gastam com esses medicamentos, bem como o volume do gasto, se reduzam ainda mais.

Por sua vez, para os medicamentos utilizados no controle do diabetes e da hipertensão, observa-se efeito redistributivo. O aumento do percentual de famílias que tiveram gasto com esses medicamentos foi maior entre as famílias de maior renda, do que entre aquelas de menor renda, no período 2002-2009. Na POF 2008-2009, os gastos com medicamentos para hipertensão e diabetes corresponderam a $4,3 \%$ do total do gasto com 
medicamentos das famílias pertencentes ao primeiro quinto de renda, enquanto esse percentual equivaleu a $13,2 \%$ entre as famílias pertencentes ao último quinto. Além disso, o gasto absoluto com medicamentos para o controle do diabetes e da hipertensão entre as famílias pertencentes ao último quinto correspondeu, respectivamente, a 15,9 e 11 vezes o gasto das famílias pertencentes ao primeiro quinto. Verifica-se assim que famílias com maior renda gastam muito mais com esses medicamentos do que as famílias de menor renda.

Não se descarta a possibilidade de que os programas de assistência farmacêutica básica do SUS tenham contribuído para esses achados. Estes programas, em que pesem as dificuldades do lado da oferta, têm um papel central para garantir o acesso a medicamentos, especialmente para as famílias mais pobres que, muitas vezes, têm neles a única forma de obtenção de medicamentos. Mesmo para a população de maior renda, é o fornecimento público que garante o acesso a tratamentos medicamentosos de alto custo 4 .

Aurea et al. 23 estudaram os programas de assistência farmacêutica do governo federal e verificaram que, entre 2005-2008, os gastos com medicamentos aumentaram e que houve um crescimento expressivo da oferta de medicamentos pertencentes aos programas de assistência farmacêutica sob responsabilidade da esfera federal, em especial de insulinas para tratamento do diabetes. A expansão do Programa Farmácia Popular do Brasil (PFPB), a partir de 2006, também pode ter contribuído parcialmente, uma vez que a alavancagem do programa ocorreu após o lançamento, em 2011, do Saúde Não Tem Preço 24.

Além da expansão da cobertura e do elenco dos programas de assistência farmacêutica do SUS, mudanças nos preços dos medicamentos também podem ter dado sua contribuição. Com a introdução dos genéricos, os preços dos medicamentos consumidos pelas famílias de menor renda podem ter apresentado redução no mesmo período. Diniz et al. 18 e Miranda et al. 25 estudaram preço e disponibilidade de medicamentos genéricos, segundo o tipo de provedor (público e privado), nas cinco regiões do Brasil, utilizando a base de dados de um estudo nacional. Os resultados não foram consistentes em todas as regiões, levando os autores a concluírem que não se poderia afirmar que os genéricos estudados eram mais caros ou mais baratos que suas contrapartes, similares ou de marca, nas cinco regiões do país. Todavia, para o Brasil, os valores foram estatisticamente significativos, mostrando média de preços maiores para os medicamentos de referência, em relação aos similares.
Dados da Pesquisa Nacional por Amostra de Domicílios, realizada pelo IBGE, em 2008, também fornecem evidências sobre a importância da assistência farmacêutica pública para a população brasileira de menor renda e também dão indicações da sua natureza progressiva: é a população de menor renda que faz mais uso do fornecimento público medicamentos. Do total da população do primeiro décimo de renda domiciliar per capita, com prescrição de algum medicamento, $64,3 \%$ tiveram acesso parcial ou total a medicamentos gratuitos; e quase a metade da população desse estrato de renda recebeu gratuitamente todos os medicamentos receitados 25. Mesmo que o acesso gratuito nem sempre seja público, tudo sugere que o SUS tenha um papel preponderante no fornecimento gratuito de medicamentos, assim como se observa em relação a outros serviços de saúde consumidos pela população de menor renda 4 .

Quando se considera a população situada no último décimo de renda domiciliar per capita, somente $15,9 \%$ obtiveram os medicamentos prescritos por meio dos programas de assistência farmacêutica do SUS 24. Entretanto, a natureza progressiva desses programas não reduz sua importância para a população de maior renda, pois o fornecimento público pode ser a única forma de acesso a medicamentos de alto custo.

Apesar da possível contribuição dos programas de assistência farmacêutica do SUS na redução das desigualdades, ainda permanecem disparidades importantes. No presente estudo, evidenciou-se, de acordo com a POF 2008-2009, que as famílias de maior renda (último quinto) gastaram, em termos absolutos, 4,5 vezes com medicamentos o valor gasto pelas famílias de menor renda (primeiro quinto).

Essas desigualdades podem refletir em dificuldades de aderência a tratamentos medicamentosos por parte da população dos estratos inferiores de renda, quando têm acesso a diagnóstico médico, mas não aos medicamentos por meio do SUS. A falta de recursos pode afetar tanto a capacidade de compra de medicamentos quanto o seguimento da posologia recomendada.

Em suma, apesar dos gastos com medicamentos terem se tornado um pouco menos regressivos, na medida em que as famílias de menor renda passaram a comprometer menor parte de sua renda na aquisição de medicamentos, permanecem desigualdades importantes. Os gastos com medicamentos são o principal componente dos gastos com saúde entre as famílias de menor renda, representando um peso importante para elas.

Tudo indica que os programas de assistência farmacêutica do SUS são a única forma de acesso 
a medicamentos de largas parcelas da população brasileira, principalmente aquela de menor renda. Dessa forma, existe um grande potencial para que as políticas públicas de assistência farmacêutica tenham um importante impacto redistributivo. A relevância da assistência farmacêutica no âmbito do SUS fica evidente ao se considerar que o acesso desigual a bens e serviços de saúde é uma das formas pelas quais se manifesta a desigualdade que marca a sociedade brasileira.
A avaliação dos gastos com a assistência farmacêutica pública, sua evolução, composição e ganhos de eficiência tornam-se informações essenciais para os gestores públicos da área de saúde. Isto permite a alocação mais eficiente dos recursos orçamentários, de forma a garantir o acesso da população aos medicamentos necessários e no tempo certo, de forma a promover a melhoria da saúde no país.

\section{Resumen}

El estudio investigó el gasto en medicamentos entre las familias brasileñas y las desigualdades relacionadas con sus ingresos, en relación con los tipos de medicamentos. Se realizó un estudio transversal, con datos de la Encuesta de Presupuestos Familiares (POF), llevada a cabo en 2002-2003 y 2008-2009. Los valores de gasto se ajustaron según el Índice de Precios al Consumo (IPCA). El índice de concentración (IC) se calculó como una medida de la desigualdad. El gasto promedio en medicamentos fue de $R \$ 53,54$ en la POF 2002-2003 y R\$59,02 en la POF 2008-2009. Los IC mostraron una concentración del gasto en las familias de mayores ingresos. La composición del gasto varía según los ingresos familiares. Las familias de menores ingresos gastan sobre todo en analgésicos, medicamentos para el resfriado y antiinflamatorios. Las familias de mayores ingresos concentran su gasto en medicamentos para la diabetes, la hipertensión y otras enfermedades del corazón. Los hogares de menores ingresos redujeron el porcentaje de su presupuesto gastado en medicamentos, pese a que este conserva una parte importante de su gasto en salud.

Utilización de Medicamentos; Economía de la Salud; Desigualdades en la Salud; Familia

\section{Colaboradores}

L. P. Garcia e L. R. S. Freitas participaram de todas as etapas da elaboração do artigo: concepção, análise, redação e revisão. A. C. Sant'Anna, L. C. G. Magalhães e A. P. Aurea colaboraram na concepção, redação e revisão da versão final do manuscrito. 


\section{Referências}

1. Brasil. Lei no 8.080 , de 19 de setembro de 1990 Dispõe sobre as condições para a promoção, proteção e recuperação da Saúde, a organização e o funcionamento dos serviços correspondentes e dá outras providências. Diário Oficial da União 1990; 20 set.

2. Ministério da Saúde. Portaria GM 3.916, de 30 de outubro de 1998. Aprova a política nacional de medicamentos. Diário Oficial da União 1998; 10 nov.

3. Ministério da Saúde. Resolução no 338, de 6 de maio de 2004. Aprova a Política Nacional de Assistência Farmacêutica. Diário Oficial da União 2004; 20 mai.

4. Silveira F, Osorio RG, Piola SF. Os gastos das famílias com saúde. In: Silveira FG, Servo LM, Menezes T, Piola SF, organizadores. Gasto e consumo das famílias brasileiras contemporâneas. Brasília: Instituto de Pesquisa Econômica Aplicada; 2006. p. 107-24.

5. Andrade MV, Lisboa MB. Determinantes dos gastos pessoais privados com saúde no Brasil. In: Silveira FG, Servo LM, Menezes T, Piola SF, organizadores. Gasto e consumo das famílias brasileiras contemporâneas. Brasília: Instituto de Pesquisa Econômica Aplicada; 2006. p. 84-106.

6. Menezes T, Campolina B, Silveira FG, Servo LMS, Piola SF. O gasto e a demanda das famílias em saúde: uma análise a partir da POF de 2002-2003. In: Silveira FG, Servo LM, Menezes T, Piola SF, organziadores. Gasto e consumo das famílias brasileiras contemporâneas. Brasília: Instituto de Pesquisa Econômica Aplicada; 2006. p. 313-43.

7. Travassos C, Viacava F, Fernandes C, Almeida CM. Desigualdades geográficas e sociais na utilização de serviços de saúde no Brasil. Ciênc Saúde Coletiva $2000 ; 5: 133-49$.

8. Silveira FG, Servo LM, Piola SF, Menezes T, organizadores. Gasto e consumo das famílias brasileiras contemporâneas. v. 1. Brasília: Instituto de Pesquisa Econômica Aplicada; 2006.

9. Silveira FG, Servo LM, Menezes T, Piola SF, organizadores. Gasto e consumo das famílias brasileiras contemporâneas. v. 2. Brasília: Instituto de Pesquisa Econômica Aplicada; 2007.

10. Paniz VMV, Fassa AG, Facchini LA, Bertoldi AD, Piccini RX, Tomasi E, et al. Acesso a medicamentos de uso contínuo em adultos e idosos nas regiões Sul e Nordeste do Brasil. Cad Saúde Pública 2008; 24:267-80.

11. Silveira FG, Osorio RG, Piola SF. Os gastos das famílias com saúde. Ciênc Saúde Coletiva 2002; 7:719-31.

12. Instituto Brasileiro de Geografia e Estatística. Pes quisa de orçamentos familiares 2008-2009 : despesas, rendimento e condições de vida. Rio de Janeiro: Instituto Brasileiro de Geografia e Estatística; 2010.

13. O’Donnell O. Measuring health inequalities in Europe: methodological issues in the analysis of survey data. Eurohealth 2009; 15:10-4.
14. Harper S, Lynch J, Meersman SC, Breen N, Davis WW, Reichman ME. An overview of methods for monitoring social disparities in cancer with an example using trends in lung cancer incidence by area-socioeconomic position and race-ethnicity, 1992-2004. Am J Epidemiol 2008; 167:889-99.

15. Kakwani N, Wagstaff A, van Doorslaer E. Socioeco nomic inequalities in health: measurement, computation, and statistical inference. J Econom 1997; 77:87-103.

16. O’Donnell O, van Doorslaer E, Wagstaff A, Lindelow M. The concentration index. Analyzing health equity using household survey data: a guide to techniques and their implementation. Washington DC: The World Bank; 2008.

17. Barros AJ, Bertoldi AD. Out-of-pocket health expenditure in a population covered by the Family Health Program in Brazil. Int J Epidemiol 2008; 37:758-65.

18. Diniz BPC, Servo LMS, Piola SF, Eirado M. Gasto das famílias com saúde no brasil: evolução e debate sobre gasto catastrófico. In: Silveira FG, Servo LM, Menezes T, Piola SF, organizadores. Gasto consumo das famílias brasileiras contemporâneas. Brasília: Instituto de Pesquisa Econômica Aplicada; 2006. p. 143-66.

19. Alonzo H, Cristiana L, Corrêa C, Zambrone F. Analgésicos, antipiréticos e antiinflamatórios não-esteroidais: dados epidemiológicos em seis centros de controle de intoxicações do Brasil. Rev Bras Toxicol 2011; 14:5-11.

20. Ramos CLJ, Targa MBM, Stein AT. Perfil das intoxicações na infância atendidas pelo Centro de Informação Toxicológica do Rio Grande do Sul (CIT/ RS), Brasil. Cad Saúde Pública 2005; 21:1134-41.

21. Agência Nacional de Vigilância Sanitária. Resolução da Diretoria Colegiada - RDC no 44, de 26 de outubro de 2010. Diário Oficial da União 2010; 28 out.

22. Agência Nacional de Vigilância Sanitária. Resolução da Diretoria Colegiada - RDC no 20, de 5 de maio de 2011. Diário Oficial da União 2011; 9 mai.

23. Aurea AP, Magalhães LCG, Garcia LP, Santos CF, Almeida RF. Programas de assistência farmacêutica do Governo Federal: estrutura atual, evolução dos gastos com medicamentos e primeiras evidências de sua eficiência, 2005-2008. Brasília: Instituto de Pesquisa Econômica Aplicada; 2011.

24. Instituto de Pesquisa Econômica Aplicada. Saúde. Políticas sociais: acompanhamento e análise. Brasília: Instituto de Pesquisa Econômica Aplicada; 2011

25. Miranda ES, Pinto CDBS, Reis ALA, Emmerick ICM Campos MR, Luiza VL, et al. Disponibilidade no setor público e preços no setor privado: um perfil de medicamentos genéricos em diferentes regiões do Brasil. Cad Saúde Pública 2009; 25:2147-58.

Recebido em 03/Set/2012

Versão final reapresentada em 15/Mar/2013 Aprovado em 26/Mar/2013 